\title{
Genetic study of the Arctic CPT1A variant suggests that its effect on fatty acid levels is modulated by traditional Inuit diet
}

\author{
Ninna Senftleber $\mathbb{1}^{1,2,3} \cdot$ Marit Eika Jørgensen ${ }^{2}$ Emil Jørsboe $\mathbb{E}^{1}{ }^{1} \cdot$ Fumiaki Imamura $\mathbb{1}^{3} \cdot$ Nita Gandhi Forouhi ${ }^{3}$. \\ Christina Lytken Larsen ${ }^{4} \cdot$ Peter Bjerregaard ${ }^{4} \cdot$ Torben Hansen $^{5,6} \cdot$ Anders Albrechtsen $^{1}{ }^{1}$
}

Received: 10 December 2019 / Revised: 29 May 2020 / Accepted: 9 June 2020 / Published online: 19 June 2020

(c) The Author(s), under exclusive licence to European Society of Human Genetics 2020

\begin{abstract}
Several recent studies have found signs of recent selection on the carnitine palmitoyl-transferase $1 \mathrm{~A}(C P T 1 A)$ gene in the ancestors of Arctic populations likely as a result of their traditional diet. CPT1A is involved in fatty acid transportation and is known to affect circulating fatty acid profiles in Inuit as does the unique traditional diet rich in marine animals. We aimed to assess which fatty acids may have driven the selection of rs80356779, a c.1436C $>\mathrm{T}$ (p.(Pro479Leu)) variant in CPT1A, by analyzing a potential interaction between the variant and traditional Inuit diet. We included 3005 genomewide genotyped individuals living in Greenland, who had blood cell membrane fatty acid levels measured. Consumption of 25 traditional food items was expressed as percentage of total energy intake. We tested for CPT1A $\times$ traditional diet interaction while taking relatedness and admixture into account. Increasing intakes of traditional diet was estimated to attenuate the effect of $479 \mathrm{~L}$ on 20:3 omega- 6 levels $(p=0.000399)$, but increase the effect of the variant on 22:5 omega3 levels $(p=0.000963)$. The 479L effect on 22:5 omega-3 more than doubled in individuals with a high intake of traditional diet (90\% percentile) compared with individuals with a low intake (10\% percentile). Similar results were found when assessing interactions with marine foods. Our results suggest that the association between traditional diet and blood cell fatty acid composition is affected by the CPTIA genotype, or other variants in linkage disequilibrium, and support the hypothesis that omega-3 fatty acids may have been important for adaptation to the Arctic diet.
\end{abstract}

Supplementary information The online version of this article (https:// doi.org/10.1038/s41431-020-0674-0) contains supplementary material, which is available to authorized users.

Ninna Senftleber

ninna.karsbaek@bio.ku.dk

1 Department of Biology, Bioinformatics Centre, University of Copenhagen, Copenhagen, Denmark

2 Steno Diabetes Center Copenhagen, Gentofte, Denmark

3 Medical Research Council Epidemiology Unit, University of Cambridge School of Clinical Medicine, Cambridge, UK

4 National Institute of Public Health, University of Southern Denmark, Copenhagen, Denmark

5 Novo Nordisk Foundation Center for Basic Metabolic Research, Faculty of Health and Medical Sciences, University of Copenhagen, Copenhagen, Denmark

6 Faculty of Health Sciences, University of Southern Denmark, Odense, Denmark

\section{Background}

For thousands of years, the Inuit ancestors have been living in a cold climate on a diet high in fat and protein, consisting of mainly fish and marine mammals [1, 2], likely increasing the need for genetic adaptation. Strong signals of positive selection have been found in the cluster of fatty acid desaturases (FADS) genes [3] and in the carnitine palmitoyl-transferase 1A (CPT1A) gene [4]. CPT1A encodes a protein involved in fatty acid transportation into the mitochondria for oxidation [5], and the c.1436C > T (NM_001876.4) variant (rs80356779) causes proline to be substituted with leucine at position 479 (NP_001867.2) in the protein sequence of CPT1A, resulting in altered enzyme activity [6]. The CPT1A rs80356779 variant is likely the causative variant [4], and in Greenland the variant is in strong linkage disequilibrium (LD) with the FADS2 rs 174570 variant, $r^{2} \approx 1$ [7]. The rs 80356779 variant is absent in individuals of European, African, and East Asian ancestry [8]. Furthermore, it is highly associated with circulating fatty acid 
composition in Inuit, suggesting that the selection pressure on the variant occurred as an adaptation to the Arctic diet $[4,7]$.

Kalaalimernit, meaning small pieces of Greenlanders, are the traditional foods of the Greenlandic Inuit [9] and consist mainly of fish and marine mammals [1]. The content of long-chain omega-3 polyunsaturated fatty acids (PUFAs) is unique for marine animals [10]. The circulating fatty acid composition in the Greenlandic population is not only affected by the CPT1A rs80356779 variant, but also by the diet, and levels of long-chain omega-3 PUFAs have been found to correlate with intake of fish and seal in Greenlandic Inuit [11]. The intake of long-chain omega-3 PUFAs from marine animals was extremely high in the traditional diet, while the intake of other PUFAs was low, which is still the case today albeit at a lesser degree [12]. Thus, the need for endogenous synthesis of the longer-chained PUFAs from shorterchained PUFAs may have been diminished, and selections on the rs80356779 variant and variants of the FADS genes may have compensated for this, as the derived alleles generally are associated with higher levels of short-chain PUFAs and lower levels of long-chain PUFAs $[3,7]$.

Results from some studies indicate that there may be a diet $\times F A D S$ interaction effect on circulating fatty acids in non-Inuit [13-15]. Interactions between diet and the CPTIA rs80356779 variant have never been assessed because the variant is nearly absent in western populations $[7,16]$. CPTIA and FADS are the genes that have been found to harbor variants that associate the strongest with fatty acid levels in Greenlandic Inuit [7]. In this study we choose not to analyze the FADS variants because the causal variant is not known and as the variants identified by Fumagalli et al. [3] are in almost perfect LD with the CPTIA variant [7], and would therefore yield similar results.

We hypothesize that selection on L479 (CPTIA) is an adaptation to the traditional Inuit diet, and studied circulating fatty acids to assess what may have been driving the selection. Specifically, we aimed to study the interaction between the rs80356779 variant and traditional Inuit diet on levels of circulating fatty acids.

By gaining insight into how the variant could have affected levels of fatty acids when the Inuit were relying exclusively on the traditional diet, we can come closer to answering this question.

\section{Methods}

\section{Study population}

We included participants from the Inuit Health in Transition cohort (2005-2010), a population-representative health survey, including a total of 3253 participants living in Greenland [17]. We excluded participants with any missing phenotype and genotype data (CPTIA genotype as well as genome-wide data) and outliers with respect to reported total energy intake, identified as outside the range of median $\pm 3 \times$ interquartile range after log transformation. We performed a sensitivity analysis, additionally excluding participants with clear under- and overreported total energy intakes, using the more strict and common-used allowable range of estimated total energy intake of $3350-17,000 \mathrm{~kJ} /$ day for men and 2100-15,000 kJ/day for women [18].

\section{Genotyping}

The individuals have previously been genotyped using the Illumina Metabo-Chip [19], an array enriched with singlenucleotide polymorphisms (SNPs) that have been found to be associated with cardiometabolic and anthropometric phenotypes in genome-wide association studies, including 196,725 SNPs in total [20]. In this study we used the genome-wide SNP chip data to infer the relatedness and population structure while the CPTIA variant was additionally selected for genotyping (LGC genomics) [7]. The Greenlandic population is genetically structured and some individuals in the study population are related. Hence, inferring relatedness and population structure, to include in the analysis, was necessary. We included autosomal SNPs, and removed SNPs with minor allele frequencies $<5 \%$ and with $>10 \%$ missing data in the whole study population.

\section{Measurements}

Information on food intake was derived from an interviewbased, semi-quantitative food frequency questionnaire, collecting information on frequency and portion size of 25 traditional foods and 43 imported foods and beverages as described in detail elsewhere [11]. Of the dietary intakes of long-chain omega-3 PUFAs, we evaluated eicosapentaenoic acid (EPA, 20:5 omega-3), docosapentaenoic acid (DPA, 22:5 omega-3), and docosahexaenoic acid (DHA, 22:6 omega-3). In this paper, we use abbreviated names of these when referring to dietary intakes. The estimated intakes of EPA, DPA, and DHA correlated with the corresponding blood measures in our study population (Pearson correlation coefficients using normalized levels of the fatty acids: 0.48 for EPA, 0.26 for DPA, and 0.33 for DHA).

Blood samples were drawn after overnight fasting; a minimum of $2 \mathrm{ml}$ full blood in a Nunc Cryo tube. Samples were stored at $-20^{\circ} \mathrm{C}$ at the study site and during transportation to Denmark, and at $-80^{\circ} \mathrm{C}$ at Steno Diabetes Center Copenhagen as well as at the Lipid Research Centre, Centre Hospitalier Université Laval, where fatty acid composition 
was measured. For samples collected in 2005 (two study sites), erythrocyte membrane phospholipids were analyzed, and for remaining samples, membrane phospholipids from all blood cell were analyzed. The same method was applied in order to isolate the cell membranes. Cell membranes of lysed cells were isolated by centrifugation and washed twice with $0.9 \% \mathrm{NaCl}$ solution. A chloroform-methanol mixture $(2: 1, \mathrm{v} /$ v) was used to extract lipids [21], and the phospholipid fraction, consisting of phosphatidylcholine, phosphatidylserine, phosphatidylinositol, phosphatidylethanolamine, and sphingomyelin, was obtained by thin layer chromatography. Phospholipid fatty acids were transmethylated and a total of 40 fatty acids were separated by capillary gas-liquid chromatography [22] and measured as a percentage of total membrane phospholipid fatty acids.

\section{Study variables}

The primary exposure is the $C P T 1 A \times$ traditional diet interaction term. Traditional foods were defined as the proportion of total energy intake [23] derived from seal meat, seal blubber, beluga and narwhale, other whales, walrus, seal offal, mattak (whale skin), cod, halibut, ammassat (capelin), trout/salmon, other fish, mussels/ shrimps/crab, dried seal and whale meat, dried fish, blubber (frozen, salted), reindeer/muskox meat, reindeer offal, razorbill, eider, other game birds, eggs from game birds, berries, dried reindeer meat, and polar bear.

Secondary dietary variables used in the interaction term were intakes of traditional marine foods, marine mammals, fish (traditional foods, not imported), EPA, EPA + DPA, $\mathrm{EPA}+\mathrm{DPA}+\mathrm{DHA}$. In the human body, EPA is a precursor of DPA, and DPA a precursor of DHA. Therefore, we anticipated that stronger interaction effects could be found using dietary intake of EPA when using blood levels of EPA as outcome, and dietary intakes of EPA + DPA for blood levels of DPA, as well as intakes of all three omega-3 PUFAs for blood levels of DHA. Furthermore, we analyzed total estimated fat and protein intake in order to assess if the interaction effects found with traditional foods were not driven by something else in the diet, and total carbohydrate, which we expected to yield somehow similar results but in an opposing direction as for traditional diet. All dietary variables were expressed as percentage of total energy intake, both to minimize the importance of any under- and overreported dietary intakes and as using this unit explained more variance in the fatty acid levels compared with using absolute intakes in $\mathrm{g} / \mathrm{d}$ while adjusting for total energy intake (data not shown).

The outcomes were the blood cell membrane phospholipid concentration of the following fatty acids: 20:5 omega-3, 22:5 omega-3, 22:6 omega-3, 18:2 omega- 6 , 20:3 omega-6, 20:4 omega-6, 22:4 omega-6, 18:1 omega-9, 20:1 omega-9, 20:0, 22:0, and 24:0, all previously found to be strongly associated with the genotype of interest, except for 20:5 omega-3 and 22:6 omega-3 [7].

\section{Statistical analysis}

We estimated interaction effects between traditional diet and rs80356779, but also performed the analysis genomewide to assess possible confounding. We applied a linear mixed model using GEMMA to account for admixture and relatedness, adjusting for age, sex, and measured cell type (erythrocytes or all blood cells) [24]

$$
y_{i}=\alpha+\beta g_{i} d_{i}+\gamma g_{i}+\delta d_{i}+\theta a_{i}+\sigma s_{i}+\omega c_{i}+\epsilon_{i}+u
$$

$\alpha$ : intercept, $\beta$ : interaction effect size, $g_{i}$ : genotype of the $i$ th individual, $d_{i}$ : intake of traditional diet of the $i$ th individual, $\gamma_{i}$ : main effect of genotype, $\delta_{i}$ : main effect of traditional diet, $\theta$ : age effect, $a_{i}$ : age of the $i$ th individual, $\sigma$ : sex effect, $s_{i}$ : sex of the $i$ th individual, $\omega$ : effect of cell type, $c_{i}$ : cell type of the $i$ th individual, $\epsilon_{i}$ : residuals, $u$ : random effects with $u N(0, G \tau)$, where $G$ is the genetic similarity matrix estimated in GEMMA based on genotype data of the included individuals and $\tau$ is a scaling parameter.

The outcomes were rank-based transformed to a standard normal distribution within each sex to achieve results that were not affected by skewed distributions.

Ancestry $\times$ environment interactions can confound gene $\times$ environment interactions [25], if both CPT1A genotype and effect of diet depend on ancestry. For the main dietary exposure, we therefore analyzed for interactions adjusting for ancestry $\times$ diet, including the interaction terms principal component $(\mathrm{PC}) 1 \times$ diet and $\mathrm{PC} 2 \times$ diet. Using the first two PCs was considered optimal for reducing the amount of inflatedness while keeping the number of parameters in the model low and adding PC3-5 $\times$ diet to the model did not reduce the amount of inflatedness further. In addition, we performed the analysis stratified for ancestry in order to assess the importance of the ancestry $\times$ diet confounder, grouping the study population into four subgroups of roughly the same size: 0-59.9\% Inuit, 60-74.9\% Inuit, 75-89.9\% Inuit, and 90-100\% Inuit. For this, we estimated separate genetic similarity matrices for each group, applying the same filters in the respective subgroups (see Genotyping for filters).

We assumed an additive effect of the rs80356779 variant on the level of each fatty acid as reported in previous studies $[4,7]$, but assessed which genetic model that best described the SNP effect on each fatty acid level by visual inspection of the data and in a linear model adjusting for age, sex, cell type measured, PC1-5, and traditional diet intake. We assumed a linear relationship between the dietary exposure and the level of fatty acids and assessed if this was reasonable by visually inspecting smoothing cubic spline plots. 
We corrected $p$ values by genomic control (GC), a method that controls for confounding effects of population structure. Unaccounted population structure could result in an inflated number of significant associations in genome-wide association analyses. Hence, the degree of inflation was estimated and the test statistics was adjusted for it when using GC [26]. We used the GC-corrected $p$ values to evaluate if interaction effects were significant. We used the GC-corrected $p$ values to evaluate if interaction effects were significant using Bonferroni for the twelve outcomes i.e., we used a level of significance of 0.00417 .

Besides including a covariate describing the type of cell measured (erythrocytes or all blood cells), we furthermore assessed if these two types of measures were comparable by estimating the main effect of the CPT1A variant on fatty acid levels in erythrocytes and all blood cells in separate linear mixed models in GEMMA.

\section{Results}

\section{Characteristics of study population}

Of the 3253 participants in the cohort, 170 had no data on blood cell fatty acid levels, 62 were not genotyped, and 16 were outliers with respect to reported total energy intake. This left 3005 for analyses. Of these, 60\% were homozygous for the derived allele, $33 \%$ were heterozygous, and $7 \%$ homozygous for the ancestral allele (derived allele frequency $=0.76$ ). The principal component analysis showed clear population structure (Supplementary Fig. 1) as previously shown [19].

Traditional diet intake varied considerably among participants (Table 1 and Supplementary Fig. 2). Of the traditional foods, marine foods contributed to the vast majority of the energy intake from traditional foods, and the energy contribution from marine mammals was considerably higher than from fish (Table 1). Correlations between the dietary exposure variables are shown in Supplementary Table 1. Analysis of the main effect of the rs80356779 variant on levels of fatty acids in erythrocytes and all blood cells respectively showed that the two types of fatty acid measures were highly comparable (Supplementary Fig. 3).

\section{Gene $\times$ diet interaction}

We tested an interaction between the rs80356779 variant and traditional Inuit diet on levels of 12 fatty acids. Of these, significant $(p<0.00417)$ or nominal significant $(p<0.05)$ interaction effects were estimated for seven fatty acids before applying GC (Supplementary Table 2). Higher intake of traditional diet was associated with a lower genotype effect on almost all of the fatty acids for which significant or nominal significant interaction effects were found (Fig. 1a-e and Supplementary Fig. 4). The exception was 22:5 omega-3: among those reporting higher intakes of traditional foods, the effect of the variant was estimated to be stronger than among those reporting lower intakes (Fig. 1f and Supplementary Fig. 4). Plots of interaction effects using non-transformed fatty acid levels are presented in Supplementary Fig. 5. See further interpretation of effect size in the "Discussion." We assessed the validity of the results in multiple ways. QQ plots from genome-wide analyses indicated potential residual confounding, especially for levels of the omega-3 fatty acids (Supplementary Fig. 6A). Further adjustment for the inflated test statistics using GC resulted in no indications of confounding in the subsequent QQ plot (Fig. 2). Six fatty acids showed significant or nominal significant effects after applying GC, with 20:3 omega-6 and 22:5 omega-3 being significant (Table 2). Second, in the genome-wide analysis adjusting for ancestry $\times$ diet, using PC $1 \times$ diet and PC2 $\times$ diet, the number of significant results reduced (Supplemental Figure 6B) with significant interactions for 20:1 omega-9 and 20:3 omega-6 as well as a nominal significant effect on 22:5 omega-3 (Supplementary Table 2). Third, we performed analyses stratified for ancestry for levels of the omega-3 fatty acids, as these showed the biggest signs of confounding in the QQ plots (Supplementary Table 3). The overall directions of the estimated interaction effects across strata were consistent, and the effects in the different strata spread around the overall effect, indicating that ancestry $\times$ diet was not an important confounder for the CPT1A interaction analysis (Supplementary Table 4 and Supplementary Figs. 7, 8).

When excluding participants based on the more strict allowable range of estimated total energy intake, very similar results were obtained as in the main analysis, both with respect to effects sizes and precision measures (Supplementary Table 5).

For most of the fatty acids, the additive model described the genotype effect most appropriately when tested using a linear model as well as visualizing the data (Supplementary Table 6 and Supplementary Fig. 9). For 20:0 and 22:0, a recessive model described the data better, and a full model for 24:0 and 22:5 omega-3. The additive model was applied for all fatty acids for simplicity. However, the full impact of the variant will be understated if the true inheritance model deviates from the assumption, and the power to detect interaction effects will be lower if a dominant or recessive model describes the genetic effect better.

The spline plots indicated it was reasonable to assume a linear relationship between traditional diet intake and the level of each fatty acid. As expected due to fewer observations in the highest intervals of traditional diet intake, 
Table 1 Characteristics of all participants and per genotype.

\begin{tabular}{|c|c|c|c|c|}
\hline \multirow[t]{2}{*}{ Characteristic } & \multirow[t]{2}{*}{ Total, $N=3005$} & \multicolumn{3}{|l|}{ rs80356779 genotypes } \\
\hline & & $\mathrm{TT}, N=1800$ & $\mathrm{TC}, N=991$ & $\mathrm{CC}, N=214$ \\
\hline Age (years) & $44.3(34.3-54.7)$ & $44.7(34.7-55.6)$ & $43.2(33.4-53.2)$ & $45.2(35.4-54.8)$ \\
\hline $\operatorname{Men}(\%)^{\mathrm{a}}$ & 44.3 & 43.4 & 45.2 & 48.1 \\
\hline$\%$ of genome of Inuit heritage ${ }^{b}$ & $77.3(61.8-92.8)$ & $88.1(75.5-100.0)$ & $65.0(50.6-76.1)$ & $47.1(30.4-59.6)$ \\
\hline \multicolumn{5}{|c|}{ Dietary intake ( $\%$ of total energy intake) } \\
\hline Traditional diet & $17.1(8.7-30.6)$ & $19.9(10.2-35.2)$ & $14.4(7.2-24.6)$ & $12.2(6.2-22.9)$ \\
\hline Traditional marine foods & $14.7(6.9-26.6)$ & $17.3(8.7-31.5)$ & $11.6(5.4-20.7)$ & $9.2(4.3-19.1)$ \\
\hline Marine mammals & $8.3(3.2-17.1)$ & $10.2(4.3-20.0)$ & $5.8(2.4-13.2)$ & $4.7(1.5-9.8)$ \\
\hline Non-marine traditional foods & $1.48(0.56-3.33)$ & $1.46(0.54-3.25)$ & $1.48(0.56-3.26)$ & $1.88(0.77-4.57)$ \\
\hline Fish & $4.66(2.18-8.88)$ & $5.15(2.41-9.85)$ & $4.16(1.77-7.79)$ & $3.99(2.01-7.47)$ \\
\hline EPA & $0.31(0.14-0.58)$ & $0.36(0.17-0.68)$ & $0.25(0.11-0.46)$ & $0.21(0.09-0.40)$ \\
\hline $\mathrm{EPA}+\mathrm{DPA}$ & $0.43(0.20-0.83)$ & $0.52(0.24-0.97)$ & $0.34(0.15-0.66)$ & $0.28(0.12-0.55)$ \\
\hline $\mathrm{EPA}+\mathrm{DPA}+\mathrm{DHA}$ & $0.75(0.34-1.38)$ & $0.88(0.42-1.60)$ & $0.59(0.27-1.12)$ & $0.49(0.24-0.97)$ \\
\hline Total fat & $33(29-38)$ & $33(29-38)$ & $33(29-37)$ & $33(28-37)$ \\
\hline Protein & $20(17-24)$ & $20(17-24)$ & $20(17-23)$ & $20(17-24)$ \\
\hline Carbohydrate & $48(40-55)$ & $47(38-55)$ & $49(42-56)$ & $49(41-56)$ \\
\hline \multicolumn{5}{|c|}{ Blood cell PL membrane level ( $\%$ of total FAs) } \\
\hline 18:1 omega-9 & $14.87(13.91-16.14)$ & $15.12(14.22-16.37)$ & $14.60(13.68-15.69)$ & $13.74(13.02-15.12)$ \\
\hline $20: 1$ omega- 9 & $0.57(0.47-0.71)$ & $0.62(0.53-0.76)$ & $0.50(0.42-0.63)$ & $0.40(0.33-0.50)$ \\
\hline $18: 2$ omega- 6 & $9.45(7.72-11.00)$ & $9.65(7.80-11.29)$ & $9.36(7.75-10.71)$ & $8.60(7.23-9.82)$ \\
\hline $20: 3$ omega- 6 & $1.17(0.83-1.62)$ & $1.13(0.79-1.62)$ & $1.27(0.90-1.68)$ & $1.06(0.83-1.31)$ \\
\hline $20: 4$ omega- 6 & $8.44(6.36-10.12)$ & $7.71(5.93-9.22)$ & $9.51(7.46-10.95)$ & $10.60(8.66-12.31)$ \\
\hline $22: 4$ omega- 6 & $0.76(0.55-1.10)$ & $0.65(0.48-0.87)$ & $0.98(0.72-1.33)$ & $1.35(0.93-1.85)$ \\
\hline $20: 0$ & $0.55(0.48-0.64)$ & $0.57(0.50-0.66)$ & $0.52(0.46-0.59)$ & $0.53(0.47-0.61)$ \\
\hline $22: 0$ & $1.81(1.56-2.11)$ & $1.89(1.58-2.18)$ & $1.74(1.53-1.98)$ & $1.73(1.56-1.93)$ \\
\hline $24: 0$ & $3.98(3.53-4.53)$ & $3.76(3.38-4.23)$ & $4.31(3.83-4.81)$ & $4.50(4.04-4.95)$ \\
\hline $20: 5$ omega- 3 & $2.14(1.17-3.64)$ & $2.44(1.32-3.91)$ & $1.76(1.00-3.14)$ & $1.67(0.97-2.97)$ \\
\hline $22: 5$ omega- 3 & 1.99 (1.54-2.32) & $1.95(1.52-2.27)$ & $2.00(1.54-2.32)$ & $2.32(1.71-2.75)$ \\
\hline $22: 6$ omega- 3 & $5.66(3.93-6.86)$ & $5.82(4.03-7.03)$ & $5.45(3.78-6.65)$ & $5.42(3.68-6.37)$ \\
\hline
\end{tabular}

$T$ derived allele, $C$ ancestral allele, $D H A$ docosahexaenoic acid, $D P A$ docosapentaenoic acid, $E P A$ eicosapentaenoic acid, $F A$ fatty acid, $P L$ phospholipid.

${ }^{\mathrm{a}}$ Reported values are median (25th-75th percentile) except for the sex distribution, which is given as proportion.

${ }^{\mathrm{b}}$ Quantification of European admixture previously published (18).

deviations from a linear tendency were observed in this part for some fatty acids (Supplementary Fig. 9).

\section{Which traditional foods are important for the interaction?}

In order to investigate what in the diet that may drive the interaction, we tested for interactions between the SNP and different dietary subgroups. Before applying GC, we observed significant and nominal significant interaction effects for roughly the same fatty acids when using traditional marine foods, marine mammals, estimated EPA, $\mathrm{EPA}+\mathrm{DPA}$, and $\mathrm{EPA}+\mathrm{DPA}+\mathrm{DHA}$ intake as exposures as for traditional diet (Supplementary Table 7). After applying GC, significant interaction effects were found on 20:1 omega-9, 20:3 omega- 6 , and 22:5 omega-3 for traditional marine foods, on 20:1 omega- 9 for marine mammals, and on 22:5 omega-3 for fish. Many of the signals disappeared using the groups of macronutrients, however, fat and carbohydrate showed similar patterns with interaction effects in opposite directions, as expected due to inverse correlation (Supplementary Table 1). All signals disappeared when using non-marine traditional foods as exposure, and fish only showed significant interaction effect on 22:5 omega-3 after GC. In order to address whether these two food groups might play a role in the interaction, we compared the interaction effects sizes. We plotted the effect sizes for each outcome for traditional non-marine 
A)

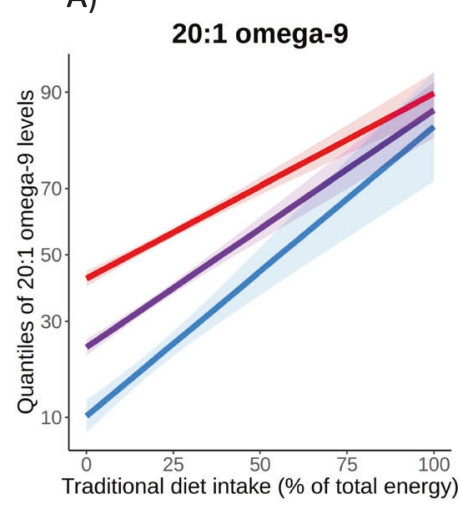

D)

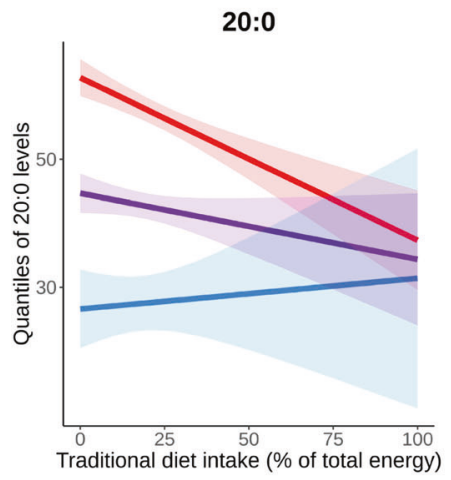

B)

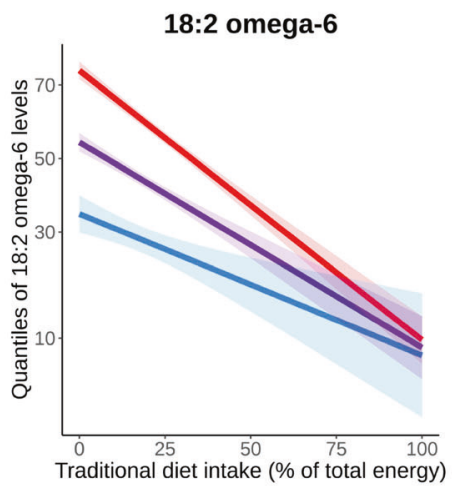

C)

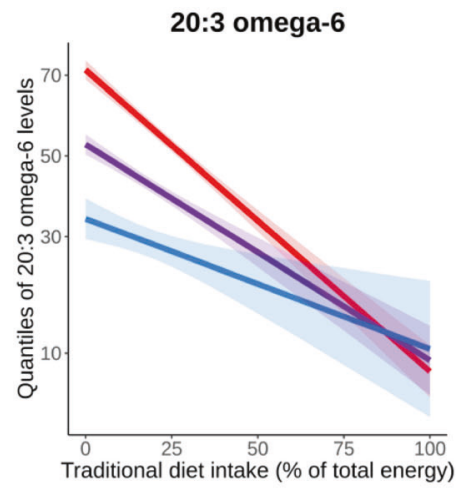

E)

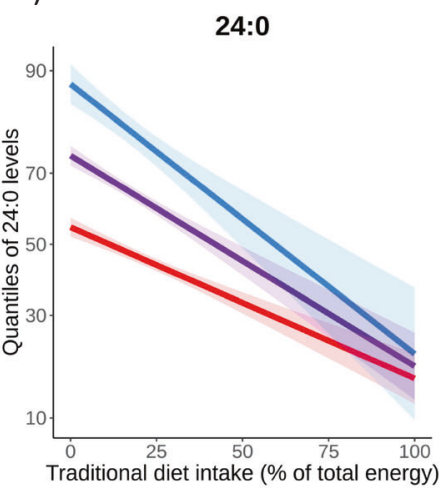

F)

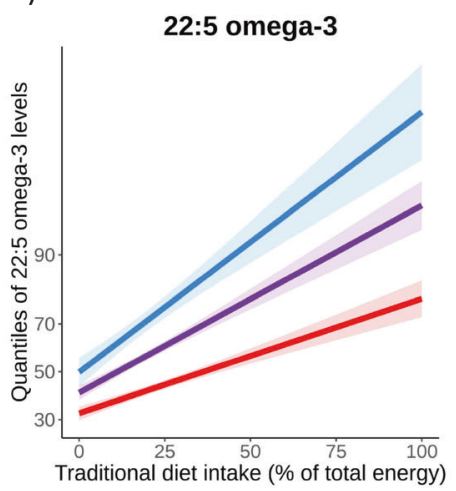

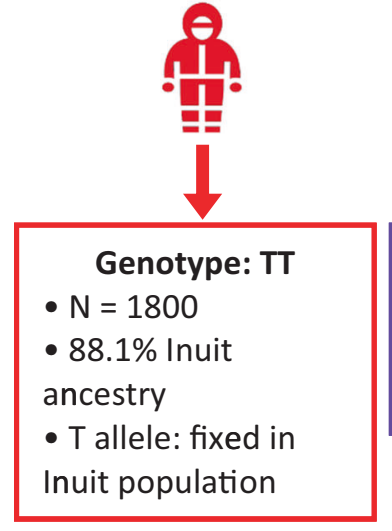

Fig. 1 Plots of the significant $(p<0.00417, \mathrm{C}$ and $\mathrm{F}$ ) and nominal significant $(p<0.05, \mathrm{~A}, \mathrm{~B}, \mathrm{D}$, and $\mathrm{E})$ interaction effects from standard interaction model adjusted for age, sex, cell type measured (erythrocytes or all blood cells), SNP effect, and diet as fixed effects and including the genetic similarity matrix as random effect. The plots are made in $\mathrm{R}$ using a model adjusted for age, sex,

foods against those for traditional marine foods, and likewise for fish against marine mammals. We observed that the effect sizes for non-marine foods were generally larger than for traditional marine foods (Supplementar Fig. 10A), and that the effect sizes for fish and marine mammals were generally in agreement (Supplementary Fig. 10B). In
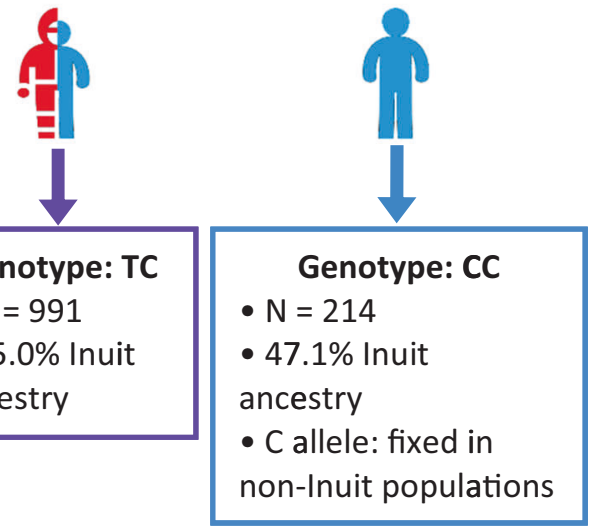

cell type measured (erythrocytes or all blood cells), SNP effect, diet, and principal component $1-5$. Inuit global ancestry proportions among the different genotypes given are medians (Table 1). The derived and ancestral allele is fixed in Inuit and non-Inuit populations respectively (7). Red: homozygous for derived allele. Purple: heterozygous. Blue: homozygous for ancestral allele. $\mathrm{T}$ derived allele, $\mathrm{C}$ ancestral allele.

general, the QQ plots before GC showed clearest indications of possible confounding for levels of the three omega3 PUFAs using the marine dietary variables as exposures, with the exception of fish intake, though applying GC resolved the inflation in all analyses (Supplementary Fig. 11). 


\section{Discussion}

We were able to include a representative and large proportion of the total Greenlandic population, namely 5.2\% [17], with detailed information on dietary intakes. Genomewide data on all participants enabled us to assess for

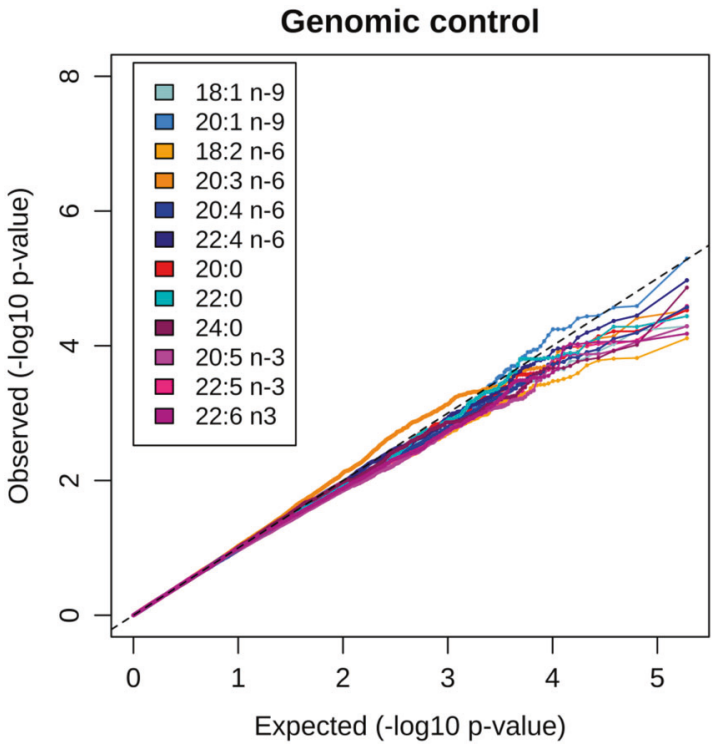

Fig. 2 QQ plots of the genome-wide association study of the CPT1A $\times$ traditional diet interaction. Analysis adjusted for age, sex, cell type measured (erythrocytes or all blood cells), SNP effect, and diet as fixed effects and including the genetic similarity matrix as random effect. $P$ values after applying genomic control unaccounted confounding factors. The Greenlandic population is unique by having a genetic variant, p.Pro479Leu in CPT1A (rs80356779), which is both common (derived allele frequency $=0.76$ ) and strongly associated with fatty acid levels. This enabled us to detect interactions with the variant despite the relatively small sample size. We found evidence that the traditional Inuit diet modulates the effect of the CPTIA rs80356779 variant on circulating levels of PUFAs. Associations between $\operatorname{diet} \times C P T 1 A$ and fatty acids from five phospholipid species were assessed jointly. Hence, we were not able to determine if the effect was evident for the individual ones. Due to LD with the variant, the interaction might also be between traditional diet and other variants like the ones found in the FADS genes [7].

\section{Risk of confounding}

We estimated the strongest interaction effects with the standard interaction model, for which QQ plots indicated potential residual confounding, which was especially evident for levels of the omega-3 PUFAs. We therefore took several approaches: (1) we applied GC and (2) performed the analyses adjusting for ancestry $\times$ diet. No substantial signs of confounding were observed when using these two approached, and our main results were based on the GCcorrected results. (3) We furthermore performed an ancestry stratified analyses, which indicated that the interaction effects were not driven by an ancestry $\times$ diet effect in the main model.
Table 2 CPT1A association result.

\begin{tabular}{|c|c|c|c|c|}
\hline \multirow[t]{2}{*}{ FA level } & \multicolumn{2}{|l|}{ Main SNP effect } & \multicolumn{2}{|l|}{$\mathrm{SNP} \times$ traditional diet } \\
\hline & SNP beta (SE) & $p$ value & $\mathrm{SNP} \times$ diet beta $(\mathrm{SE})$ & GC $p$ value \\
\hline 18:1 omega-9 & $-3.84 \mathrm{E}-01(4.37 \mathrm{E}-02)$ & $6.06 \mathrm{E}-18$ & $2.10 \mathrm{E}-03(1.89 \mathrm{E}-03)$ & $2.94 \mathrm{E}-01$ \\
\hline 20:1 omega-9 & $-4.52 \mathrm{E}-01(3.68 \mathrm{E}-02)$ & $1.88 \mathrm{E}-32$ & $4.02 \mathrm{E}-03(1.49 \mathrm{E}-03)$ & $7.38 \mathrm{E}-03$ \\
\hline $18: 2$ omega- 6 & $-3.68 \mathrm{E}-01(3.60 \mathrm{E}-02)$ & $2.20 \mathrm{E}-23$ & $4.45 \mathrm{E}-03(1.53 \mathrm{E}-03)$ & $1.18 \mathrm{E}-02$ \\
\hline $20: 3$ omega- 6 & $-3.18 \mathrm{E}-01(3.71 \mathrm{E}-02)$ & $3.12 \mathrm{E}-17$ & $5.67 \mathrm{E}-03(1.52 \mathrm{E}-03)$ & $3.99 E-04$ \\
\hline $20: 4$ omega- 6 & $3.07 \mathrm{E}-01(3.93 \mathrm{E}-02)$ & $1.23 \mathrm{E}-14$ & $-2.69 \mathrm{E}-03(1.66 \mathrm{E}-03)$ & $1.00 \mathrm{E}-01$ \\
\hline $22: 4$ omega- 6 & $4.41 \mathrm{E}-01(3.66 \mathrm{E}-02)$ & $1.94 \mathrm{E}-31$ & $-9.39 \mathrm{E}-04(1.50 \mathrm{E}-03)$ & $5.06 \mathrm{E}-01$ \\
\hline 20:0 & $-4.03 \mathrm{E}-01(4.19 \mathrm{E}-02)$ & $5.03 \mathrm{E}-21$ & $5.08 \mathrm{E}-03(1.84 \mathrm{E}-03)$ & $1.63 \mathrm{E}-02$ \\
\hline $22: 0$ & $-4.32 \mathrm{E}-01(3.88 \mathrm{E}-02)$ & $3.08 \mathrm{E}-27$ & $2.94 \mathrm{E}-03(1.67 \mathrm{E}-03)$ & $8.98 \mathrm{E}-02$ \\
\hline $24: 0$ & $4.09 \mathrm{E}-01(3.97 \mathrm{E}-02)$ & $1.02 \mathrm{E}-23$ & $-4.73 \mathrm{E}-03(1.67 \mathrm{E}-03)$ & $1.39 \mathrm{E}-02$ \\
\hline $20: 5$ omega-3 & $8.68 \mathrm{E}-02(3.75 \mathrm{E}-02)$ & $2.06 \mathrm{E}-02$ & $3.58 \mathrm{E}-03(1.47 \mathrm{E}-03)$ & $7.42 \mathrm{E}-02$ \\
\hline $22: 5$ omega- 3 & $3.63 \mathrm{E}-01(4.23 \mathrm{E}-02)$ & $3.47 \mathrm{E}-17$ & $8.06 \mathrm{E}-03(1.79 \mathrm{E}-03)$ & $9.63 E-04$ \\
\hline $22: 6$ omega-3 & $-4.22 \mathrm{E}-02(4.06 \mathrm{E}-02)$ & $2.99 \mathrm{E}-01$ & $1.61 \mathrm{E}-03(1.78 \mathrm{E}-03)$ & $4.91 \mathrm{E}-01$ \\
\hline
\end{tabular}

Analysis of the main and interaction effect of the SNP on levels of each fatty acid. Analysis for the main SNP effect was adjusted for age, sex, and cell type measured (erythrocytes or all blood cells) as fixed effects and including the genetic similarity matrix as random effect. Analysis for the SNP $\times$ diet interaction effect was adjusted for age, sex, cell type measured (erythrocytes or all blood cells), SNP effect, and diet as fixed effects and including the genetic similarity matrix as random effect. SNP beta and SNP $\times$ diet beta is the estimated effect size in standard deviations. GC $p$ value: $p$ values after applying genomic control. Significant interaction effects are marked with bold $(p<0.00417)$. 


\section{Effect size of the interaction}

We considered the estimated interaction effect to be large. For example, the estimated effect of the derived allele (L479) on 22:5 omega-3 levels was 2.3 times larger at an intake of 45 energy percent from traditional foods (corresponding to the $90 \%$ percentile, beta[SNP] $=0.58 \mathrm{SD}$ ) compared with the effect at an intake of 4.4 energy percent (the $10 \%$ percentile, beta $[\mathrm{SNP}]=0.24 \mathrm{SD}$ ). Hence, the interaction effect was quite large. A meta-analysis including cohorts of different populations suggests that $1 \mathrm{SD}$ increase in circulating levels of 22:5 omega-3 is associated with a $10 \%$ risk reduction of fatal coronary heart disease [27]. The model predicted the effect of L479 on 20:3 omega-6 levels to be 2.2 times lower at an intake of 45 energy percent compared with at 4.4 energy percent.

\section{Which fatty acids were important for the selection of rs80356779?}

We can speculate on explanations for the directions of the observed interaction effects. Omega-6 PUFAs are rare in traditional foods and having a genetic variant that favors higher levels of these would be beneficial when depending on this diet, as they are essential to ingest for humans [28]. L479 was associated with higher levels of 20:3 omega-6, however, we observed that the effect of the SNP was lower at higher intakes of traditional diet (Fig. 2c). If selection occurred to favor 20:3 omega-6, we would expect the genotype effect at high intakes of traditional foods to be larger than at low intakes (Supplementary Fig. 12). The effect of L479 on levels of the remaining fatty acids attenuated as well at higher intakes of traditional diet, with the exception of 22:5 omega-3, indicating that they did not play a major role in selection of the $C P T 1 A$ variant. The fact that we estimated larger genotype effects at higher intakes of traditional foods for levels of 22:5 omega-3 indicates that the omega-3 fatty acid might have been important for the selection of the variant(s). We observed that the derived allele was associated with lower levels of 20:5 omega-3 and 22:5 omega-3 as well as weaker positive associations with higher intakes of traditional diet. The long-chain omega-3 PUFAs are essential for humans [28] and have many potential health benefits [29] but have also been hypothesized to increase bleeding risk in both Inuit and western populations [30, 31]. Therefore, the extremely high intake of long-chain omega-3 PUFAs from a 100\% traditional Inuit diet could potentially be harmful. Minimizing levels of long-chain omega-3 PUFA levels could therefore have been relevant for survival of the Inuit, and it could explain the observed interaction between Inuit diet and the selected rs80356779 variant on 22:5 omega-3 levels. However, these potential benefits might have outweighed the increased risk of infant mortality that has also been associated with the variant [32].

\section{Which foods are driving the interaction?}

Both diet and the CPT1A rs80356779 variant are strongly associated with fatty acid levels. As the CPTIA gene is involved in fatty acid metabolism, the obvious environmental candidate for any interactions with the variant is dietary factors. Other candidates such as a high physical activity level, which is also part of the traditional hunter lifestyle of the Inuit, did not correlate with traditional diet in the study population. Therefore, it is unlikely to be a better proxy for what is driving the interaction. However, the intake of traditional diet still serves as a proxy of what is actually interacting with the rs80356779 variant, and the $\mathrm{SNP} \times$ diet interaction might be driven by the marine component of the diet. The effect sizes could indicate that non-marine traditional foods were of importance as well, and maybe to a larger degree than marine foods, as the effect sizes were larger (Supplementary Fig. 10A). However, we need to interpret the plot with caution, as the fitted line heavily depended on the two outermost points. Though traditional fish may not have been the driver for interaction observed for marine foods, we cannot conclude that fish has not been of importance, as it yielded effects of the same sizes as for marine mammals. The lack of significant results for fish and non-marine traditional foods could likely be due to lower intakes and hence relatively more uncertainty of estimates.

\section{What is the consequence of the selection in today's Greenland?}

The Greenlandic population is currently experiencing a westernization of their diet [12]. The interaction we observed could be important to consider when addressing health consequences of the current westernization. Carriers of the rs80356779 allele (L479) might need to consume more marine foods in order to achieve the optimal levels of 20:5 omega-3 and 22:5 omega-3 compared with noncarriers. Furthermore, it is possible that other genetic variants affect the individual response to the more westernized diet, making it relevant to further investigate the role of gene $\times$ diet interactions in obesity and metabolic diseases in the Greenlandic population, as several relevant genetic variants exists [33]. For example, the intake of sugar, especially from soft drinks, is high in Greenland [12], and potential effects of sugars like high-fructose corn sirup [34] could be different in Inuit. This may become evident in the years to come, after high-fructose corn sirup was allowed in the EU in 2017 and is widely used in soft drinks in the US [35]. More personalized dietary advice could be of future 
interest along with the need to identify optimal intakes and circulating levels of long-chain PUFAs in this population.

\section{Previous studies of FADS $\times$ diet interactions}

Previous studies have assessed $F A D S \times$ diet interactions on circulating fatty acid levels. Takkunen et al. [13] studied the interaction effect between FADS1 (rs174550) and marine PUFA intake on different erythrocyte fatty acids in Finnish individuals. Similar to our results, they found a nominally significant interaction effect on 20:5 omega-3 levels. Juan et al. [14] analyzed interactions between FADS1 (rs174546 in complete LD with rs174550 used by Takkunen et al. [13]) and dietary EPA and DHA intakes on plasma 20:5 omega-3 proportions in Americans. They found nominally significant interactions for both FADS $1 \times$ EPA intake and FADS $1 \times$ DHA intake $(p=0.02$ and 0.01 respectively, adjusted for sex and the top four PCs; number of tests = 25). Lemaitre et al. [36] assessed interactions between a FADS2 variant (rs1535) and fish consumption ( $\geq 0.6$ vs. $<0.6$ servings/week) in participants of the consortium of cohorts of European ancestry, but only for plasma phospholipid levels of 20:5 omega-3 and 22:6 omega-3, and did not find a significant interaction. This is consistent with our findings, though there was no main effect of the CPTIA genotype on 22:6 omega-3 levels in the Greenlandic population. The consistency may be meaningful given the caveats of comparing the potential interaction effects across different populations for the following reasons. First, interactions between different genes and different dietary exposures were assessed and in populations of different ethnicity. Second, the three studies used different SNPs in the FADS genes than the one in the Greenlandic cohort, and the LD between the causal unknown SNP and the one used might be different in the cohorts.

\section{Conclusion}

Our results provide evidence that the traditional diet modify the effect of the rs 80356779 variant on circulating levels of PUFAs. Combining genotype information with levels of long-chain omega-3 PUFA will likely be a better biomarker of marine food intake than solely the levels. Furthermore, the potential interaction between traditional Inuit diet and CPTIA genotype should be considered when addressing consequences of the westernization of the diet of Inuit populations. The intakes of traditional marine foods and marine mammals were driving the signal for interaction, though the other traditional foods may be involved in the interaction. The long-chain omega-3 PUFAs might have been important for driving the selection of the rs80356779 variant, maybe to minimize possible harmful effects of the extremely high intake of these that the Inuit have had. Based on our study, we suggest that the high intake of marine foods of the ancestral Inuit and the consequently high level of circulating long-chain omega-3 PUFAs is currently the best candidate for explaining why L479 in CPTIA underwent positive selection.

\section{Data availability}

The Greenlandic Metabochip-genotype data are deposited in the European Genome-phenome Archive (https://www.ebi. ac.uk/ega/home) with the accession EGAS00001002641.

Acknowledgements We would like to direct our acknowledgement to the staff and participants of the Inuit Health in Transition cohort facilitating this study as well as the Popgen group at the Bioinformatics Centre for a very helpful manuscript discussion.

Funding NKS and AA are funded by the Lundbeck foundation (R215-2015-4174). NGF and FI acknowledge funding from the Medical Research Council Epidemiology Unit MC_UU_12015/5. NGF also acknowledges NIHR Biomedical Research Centre Cambridge: Nutrition, Diet, and Lifestyle Research Theme (IS-BRC1215-20014).

Author contributions NKS and AA designed the project with input from MEJ, FI, and NGF; NKS undertook the statistical analyses with help from EJ; NKS wrote the majority of the manuscript with critical input from AA, FI, and MEJ. NKS, MEJ, EJ, FI, NGF, CLL, PB, TH, and AA read, commented, and approved the manuscript.

\section{Compliance with ethical standards}

Conflict of interest The authors declare that they have no conflict of interest.

Publisher's note Springer Nature remains neutral with regard to jurisdictional claims in published maps and institutional affiliations.

\section{References}

1. Pars T, Osler M, Bjerregaard P. Contemporary use of traditional and imported food among Greenlandic Inuit. Arctic. 2001;54:22-31.

2. Raghavan M, DeGiorgio M, Albrechtsen A, Moltke I, Skoglund $\mathrm{P}$, Korneliussen TS, et al. The genetic prehistory of the New World Arctic. Science. 2014;345:1255832.

3. Fumagalli M, Moltke I, Grarup N, Racimo F, Bjerregaard P, Jørgensen ME, et al. Greenlandic Inuit show genetic signatures of diet and climate adaptation. Science. 2015;349:1343-7.

4. Skotte L, Koch A, Yakimov V, Zhou SR, Søborg B, Andersson $\mathrm{M}$, et al. CPT1A missense mutation associated with fatty acid metabolism and reduced height in Greenlanders. Circ-Cardiovasc Genet. 2017;10:1-9.

5. McGarry JD, Brown NF. The mitochondrial carnitine palmitoyltransferase system-from concept to molecular analysis. Eur J Biochem. 1997;244:1-14.

6. Brown NF, Mullur RS, Subramanian I, Esser V, Bennett MJ, Saudubray JM, et al. Molecular characterization of L-CPT I deficiency in six patients: insights into function of the native enzyme. J Lipid Res. 2001;42:1134-42. 
7. Andersen MK, Jørsboe E, Sandholt CH, Grarup N, Jørgensen ME, Færgeman NJ, et al. Identification of novel genetic determinants of erythrocyte membrane fatty acid composition among Greenlanders. PLoS Genet. 2016;12:1-19.

8. Rajakumar C, Ban MR, Cao HN, Young TK, Bjerregaard P, Hegele RA. Carnitine palmitoyltransferase IA polymorphism $\mathrm{P} 479 \mathrm{~L}$ is common in Greenland Inuit and is associated with elevated plasma apolipoprotein A-I. J Lipid Res. 2009;50:1223-8.

9. Sowa F. Kalaalimernit: the Greenlandic taste for local foods in a globalised world. Polar Rec. 2015;51:290-300.

10. Linder M, Belhaj N, Sautot P, Arab Tehrany E. From Krill to Whale: an overview of marine fatty acids and lipid compositions. OCL. 2010;17:194-204.

11. Jeppesen C, Jørgensen ME, Bjerregaard P. Assessment of consumption of marine food in Greenland by a food frequency questionnaire and biomarkers. Int J Circumpolar Health. 2012;71: $1-8$.

12. Jeppesen C, Bjerregaard P. Consumption of traditional food and adherence to nutrition recommendations in Greenland. Scand J Public Health. 2012;40:475-81.

13. Takkunen MJ, de Mello VD, Schwab US, Kuusisto J, Vaittinen $\mathrm{M}$, Agren JJ, et al. Gene-diet interaction of a common FADS1 variant with marine polyunsaturated fatty acids for fatty acid composition in plasma and erythrocytes among men. Mol Nutr Food Res. 2016;60:381-9.

14. Juan J, Huang HY, Jiang X, Korat AVA, Song MY, Sun Q, et al. Joint effects of fatty acid desaturase 1 polymorphisms and dietary polyunsaturated fatty acid intake on circulating fatty acid proportions. Am J Clin Nutr. 2018;107:826-33.

15. Smith CE, Follis JL, Nettleton JA, Foy M, Wu JHY, Ma YY, et al. Dietary fatty acids modulate associations between genetic variants and circulating fatty acids in plasma and erythrocyte membranes: Meta-analysis of nine studies in the CHARGE consortium. Mol Nutr Food Res. 2015;59:1373-83.

16. Clemente FJ, Cardona A, Inchley CE, Peter BM, Jacobs G, Pagani $\mathrm{L}$, et al. A selective sweep on a deleterious mutation in CPT1A in Arctic populations. Am J Hum Genet. 2014;95:584-9.

17. Bjerregaard $P$. Inuit health in transition-Greenland survey 2005-10. Population sample and survey methods. SIF writings on Greenland, vol 19. 2nd ed. Copenhagen: National Institute of Public Health; 2011.

18. Willett W. Issues in analysis and presentation of dietary data: nutritional epidemiology. 3rd ed. Oxford: Oxford University Press; 2013. p. 305-32.

19. Moltke I, Fumagalli M, Korneliussen TS, Crawford JE, Bjerregaard P, Jørgensen ME, et al. Uncovering the genetic history of the present-day Greenlandic population. Am J Hum Genet. 2015;96:54-69.

20. Voight BF, Kang HM, Ding J, Palmer CD, Sidore C, Chines PS, et al. The Metabochip, a custom genotyping array for genetic studies of metabolic, cardiovascular, and anthropometric traits. PLoS Genet. 2012;8:1-12.
21. Rudkowska I, Ouellette C, Dewailly E, Hegele RA, Boiteau V, Dube-Linteau A, et al. Omega-3 fatty acids, polymorphisms and lipid related cardiovascular disease risk factors in the Inuit population. Nutr Metab. 2013;10:1-9.

22. Da Silva MS, Julien P, Perusse L, Vohl MC, Rudkowska I. Natural rumen-derived trans fatty acids are associated with metabolic markers of cardiac health. Lipids. 2015;50:873-82.

23. Willett WC, Howe GR, Kushi LH. Adjustment for total energy intake in epidemiologic studies. Am J Clin Nutr. 1997;65:1220-8.

24. Zhou X, Stephens M. Genome-wide efficient mixed-model analysis for association studies. Nat Genet. 2012;44:821-4.

25. Keller MC. Gene $\times$ environment interaction studies have not properly controlled for potential confounders: the problem and the (Simple) solution. Biol Psychiatry. 2014;75:18-24.

26. Devlin B, Roeder K. Genomic control for association studies. Biometrics. 1999;55:997-1004.

27. Del Gobbo LC, Imamura F, Aslibekyan S, Marklund M, Virtanen JK, Wennberg M, et al. Omega-3 polyunsaturated fatty acid biomarkers and coronary heart disease pooling project of 19 cohort studies. JAMA Intern Med. 2016;176:1155-66.

28. Di Pasquale MG. The essentials of essential fatty acids. J Diet. 2009;6:143-61.

29. Hooper L, Abdelhamid A, Ajabnoor S, Brainard J, Brown T, Hanson S, et al. Set of systematic reviews of RCTs on the health effects of omega 3 polyunsaturated fats in adults. World Health Organization; 2017.

30. Dyerberg J, Bang HO. Haemostatic function and platelet polyunsaturated fatty acids in Eskimos (Reprinted from Lancet, vol 2, pg 433, 1979). Nutrition. 1995;11:475.

31. Jeansen S, Witkamp RF, Garthoff JA, van Helvoort A, Calder PC. Fish oil LC-PUFAs do not affect blood coagulation parameters and bleeding manifestations: analysis of 8 clinical studies with selected patient groups on omega-3-enriched medical nutrition. Clin Nutr. 2018:37:948-57.

32. Sinclair GB, Collins S, Popescu O, McFadden D, Arbour L, Vallance HD. Carnitine palmitoyltransferase I and sudden unexpected infant death in British Columbia First Nations. Pediatrics. 2012;130:e1162-9.

33. Andersen MK, Hansen T. Genetics of metabolic traits in Greenlanders: lessons from an isolated population. J Intern Med. 2018;284:464-77.

34. Simopoulos AP. Dietary omega-3 fatty acid deficiency and high fructose intake in the development of metabolic syndrome, brain metabolic abnormalities, and non-alcoholic fatty liver disease. Nutrients. 2013;5:2901-23.

35. ICF Consulting Services Ltd. Consumption and impact of high fructose syrups. Luxemborg: European Union; 2018.

36. Lemaitre RN, Tanaka T, Tang WH, Manichaikul A, Foy M, Kabagambe EK, et al. Genetic loci associated with plasma phospholipid n-3 fatty acids: a meta-analysis of genome-wide association studies from the CHARGE Consortium. Plos Genet. 2011;7:1-12. 